Mens

revue d'histoire intellectuelle de l'Amérique française

John S. Moir. Christianity in Canada. Historical Essays. Édition préparée par Paul Laverdure. Yorkton, Redeemer's Voice Press, 2002. $188 \mathrm{p}$.

John S. Moir. Early Presbyterianism in Canada. Essays. Édition préparée par Paul Laverdure. Gravelbourg, Gravelbooks, 2003. 266 p.

Paul Laverdure. Sunday in Canada. The Rise and Fall of the Lord's Day. Yorkton, Gravelbooks, 2004. 253 p.

Jozef De Vocht, CSSR. Eternal Memory! Father Achiel Delaere (1868-1939). The First Eastern Rite Redemptorists and Canada's Ukrainian Catholic Church. Yorkton, Gravellbooks, 2005. 327 p.

\title{
Christian Roy
}

Volume 7, numéro 2, printemps 2007

URI : https://id.erudit.org/iderudit/1024136ar

DOI : https://doi.org/10.7202/1024136ar

Aller au sommaire du numéro

Éditeur(s)

Centre de recherche en civilisation canadienne-française

ISSN

1492-8647 (imprimé)

1927-9299 (numérique)

Découvrir la revue

Citer ce compte rendu

Roy, C. (2007). Compte rendu de [John S. Moir. Christianity in Canada. Historical Essays. Édition préparée par Paul Laverdure. Yorkton, Redeemer's Voice Press, 2002. 188 p. / John S. Moir. Early Presbyterianism in Canada. Essays. Édition préparée par Paul Laverdure. Gravelbourg, Gravelbooks, 2003. 266 p. / Paul Laverdure. Sunday in Canada. The Rise and Fall of the Lord's Day. Yorkton, Gravelbooks, 2004. 253 p. / Jozef De Vocht, CSSR. Eternal Memory! Father Achiel Delaere (1868-1939). The First Eastern Rite Redemptorists and Canada's Ukrainian Catholic Church. Yorkton, Gravelbooks, 2005. 327 p.] Mens, 7(2),

359-367. https://doi.org/10.7202/1024136ar d'utilisation que vous pouvez consulter en ligne. 
John S. Moir. Christianity in Canada. Historical Essays. Édition préparée par Paul Laverdure. Yorkton, Redeemer's Voice Press, 2002. 188 p.

John S. Moir. Early Presbyterianism in Canada. Essays. Édition préparée par Paul Laverdure. Gravelbourg, Gravelbooks, 2003. 266 p.

Paul Laverdure. Sunday in Canada. The Rise and Fall of the Lord's Day. Yorkton, Gravelbooks, 2004. $253 \mathrm{p}$.

Jozef De Vocht, CSSR. Eternal Memory! Father Achiel Delaere (1868-1939). The First Eastern Rite Redemptorists and Canada's Ukrainian Catholic Church. Yorkton, Gravelbooks, 2005. 327 p.

Récemment élu président de la Société canadienne d'histoire ecclésiastique - fonction qu'occupa aussi son directeur de thèse, John S. Moir, Paul Laverdure a contribué à cette discipline d'une façon qui trompe les attentes. FrancoOntarien d'origine, il s'est d'abord spécialisé dans l'histoire du protestantisme anglo-saxon, mais a surtout marqué celle du catholicisme anglo-canadien - cette " troisième solitude", selon l'expression de Moir, faisant référence à son statut doublement minoritaire, en marge du Canada anglo-protestant d'une part et de l'Église franco-catholique d'autre part. Le premier livre de Laverdure, Redemption and Renewal. The Redemptorists of English Canada 1834-1994, est une histoire officielle de cet ordre religieux, parue à Toronto en 1996. La même année, il quitte Montréal pour s'occuper des archives des Rédemptoristes à Yorkton en Saskatchewan, siège de la branche de rite oriental de cet ordre connu au Québec comme responsable du pèlerinage de Sainte-Anne-de-Beaupré. Celleci fut créée il y a un siècle pour répondre aux besoins pasto- 
raux très particuliers des immigrants ukrainiens d'obédience romaine, mais farouchement nationalistes, tiraillés entre les sollicitations de l'irréligion, de l'Église orthodoxe dont ils gardaient rites et coutumes, du prosélytisme protestant et d'un foisonnement de schismes et de sectes dans les conditions très rudes de la colonisation des Prairies. C'est de là pourtant que ce modèle d'acculturation des ordres religieux latins dans des communautés de rite grec s'est propagé jusque dans le vieux pays et à travers la diaspora ukrainienne internationale. Or il cette expérience audacieuse était le fait de missionnaires flamands appelés au Canada par $\mathrm{M}^{\mathrm{gr}}$ Langevin de SaintBoniface au Manitoba. Moyennant des stages en Galicie, ceuxci missionnaires catholiques durent s'immerger dans la culture slave et passer au rite byzantin, alors très mal connu en Occident. Le premier à faire le saut fut le Père Achiel Delaere, et c'est pour marquer le centenaire de ce point tournant en 2006 que les Rédemptoristes canadiens ont parrainé la traduction du néerlandais de sa biographie par le Père Jozef De Vocht, parue en Belgique il y a un demi-siècle. Cette nouvelle édition, richement illustrée de documents d'archives par les soins de Paul Laverdure, lève le voile sur un pan méconnu et haut en couleurs de l'histoire du christianisme au Canada, pour montrer sous un angle inédit l'épineux problème qu'a constitué pour l'Église romaine la perpétuation d'identités ethniques particularistes (qu'elles soient slaves, germaniques ou latines - y compris francophones) sous couvert d'un catholicisme universaliste, qu'elle cherchait de son côté à acclimater au contexte anglophone de l'Amérique du Nord.

Laverdure s'est ainsi établi à son compte comme historien privé et éditeur de plusieurs livres dans le domaine de l'histoire du christianisme canadien, qui ont souvent joui d'un succès d'estime dans le monde de l'édition en Saskatchewan. Certains d'entre eux méritent sans doute d'être plus largement connus de la communauté historienne, parmi laquelle un 
Michael Bliss a pu qualifier d'« exemplaire » le livre récemment tiré par Laverdure de sa thèse de doctorat sur l'observance du dimanche au Canada, dirigée par John S. Moir à l'Université de Toronto. $\mathrm{Y}$ ayant lui-même eu Donald Creighton comme directeur de thèse, Moir fait figure de pionnier de l'histoire de la religion au Canada et Laverdure a tenu à publier deux recueils d'articles de son maître, sélectionnés en consultation avec lui, dont plusieurs étaient demeurés inédits ou devenus inaccessibles. Même si je me dois de signaler aussi au passage la somptueuse édition anglaise, établie par Paul Laverdure avec la collaboration de Jacqueline et John S. Moir de deux ouvrages introuvables du missionaire arctique français Émile Petitot (Travels Around Great Slave and Great Bear Lakes 1862-1882, Toronto, Champlain Society, 2005, 454 p.), je voudrais surtout livrer ici un aperçu des perspectives qu'offrent sur l'histoire religieuse du Canada ces trois livres, disponibles chez l'éditeur saskatchewanais Gravelbooks.

Né en 1926, le Torontois John Sargent Moir a d'abord consacré un mémoire de maitrise au méthodisme réformateur et loyaliste du pédagogue et journaliste Egerton Ryerson au Haut-Canada, avant de se tourner pour son doctorat vers les relations entre les Églises et l'État au Canada-Ouest, vues au prisme de la question scolaire et de celle des réserves du clergé. S'il parle alors de la tension entre un « confessionnalisme centrifuge » et un " nationalisme centripète », il précisera plus tard qu'en l'absence de religion civile soudant une nation unitaire, les Églises ont chacune fourni aux Canadiens une identité transcontinentale de rechange qui faisait contrepoids aux identités régionales plus vivaces des "sociétés distinctes » coexistant au sein de la Confédération (CiC, p. 157). Moir en effet ne perd jamais de vue les liens étroits unissant les questions d'identité religieuse et d'identité politique, même lorsqu'il se concentre sur une confession comme le presbytérianisme, dont il a publié une histoire en 1974, deux ans après l'avoir re- 
jointe (venant de l'Église unie). Selon lui, nonobstant la division linguistique, l'étroite gamme d'affiliations religieuses des Canadiens témoigne d'une relative homogénéité qui "peut être vue comme une double défense - contre les défis de la nature et contre la menace constante d'absorption culturelle et politique par les États-Unis. »C'est ce que Moir appelle, à la suite de Robert Handy, " "churchliness" - a national habit of supporting only a few major denominations and an avoidance of religious pluralism as it is known in the United States » (CiC, p. 137). Cela tient selon lui à l'attitude contrerévolutionnaire longtemps partagée par les Canadiens français et anglais : dépourvus d'identité forte et dispersés dans une nature menaçante, les uns et les autres ont cherché à s'adosser aux traditions les reliant à leurs métropoles respectives, notamment sur le plan ecclésiastique, puisqu'une part importante du clergé en est longtemps provenue. De là l'interférence entre des problématiques importées des Églisesmères et les conditions coloniales qui leur demeuraient irréductibles. Ainsi, l'idéal érastien d'Église nationale d'État ou " établie » se heurtait-il au pluralisme confessionnel de facto de l'Amérique du Nord britannique. Cela a commencé avec l'anomalie du double establishment de la Nouvelle-France, conquise au prix de la reconnaissance de l'Église catholique canadienne - qui retrouvait, avec le départ de la France, l'autonomie confisquée par l'absolutisme gallican en 1663, tandis que l'Église anglicane demeurait au Canada une branche de l'État britannique $(C i C$, p. 14). Au Haut-Canada même, des colons américains n'ont pas tardé à se mêler aux émigrés loyalistes, et le soupçon de déloyauté a longtemps pesé sur leurs confessions transfrontalières aux structures plus légères - jusqu'à ce que les événements de 1812 et de 1837-38 leur donnent l'occasion de rivaliser de loyalisme britannique avec l'Église anglicane établie. Son champion, l'évêque John Strachan, la croyait néanmoins destinée à devenir l'Église nationale du Canada 
anglais ; il suffisait pour cela selon lui que l'État lui gagnât les autres protestants en dotant leur clergé de généreuses cures, selon le principe de patronage de l'establishment. Or les limites inhérentes à ce modèle de rapport entre l'Église et l'État finirent par le rendre impraticable, malgré la tentative du CanadaUni de l'étendre sélectivement aux Églises qui s'y prêtaient, tandis que d'autres Églises persistaient à s'y refuser par principe "volontariste ». C'est dans ce contexte qu'il faut situer l'abolition des réserves du clergé en 1854 dans l'unique texte législatif canadien énonçant une séparation de l'Église et de l'État. Cette séparation était d'ailleurs favorisée par l'avantage relatif des confessions non conformistes décentralisées sans liens organiques avec l'Ancien Monde parmi des populations coloniales mobiles et dispersées, dont les besoins d'encadrement religieux étaient plus aisément satisfaits par le nomadisme du circuit de prédicateurs que par la structure sédentaire de la paroisse. Leur contestation de l'establishment stato-ecclésial procédait pourtant de positions théocratiques radicales qui ne prenaient pas toujours cette forme démocratique, puisqu'il faut notamment compter parmi elles l'ultramontanisme, ainsi que Moir a su le voir : "Anti-Erastianism was the bed-rock of the Romantics' opposition to statism, whether voiced in Edinburgh, Oxford, Rome or Paris, yet antiErastianism per se is not necessarily voluntarist » (EPiC, p. 16).

Le volontarisme des confessions non conformistes a même pu jouer le rôle d'une sorte de nouvel érastianisme à la faveur de leur convergence autour de ce que Moir appelle un " protestantisme omnibus ", basé sur une théologie victorienne de l'incarnation du Royaume de Dieu dans l'histoire collective par les moyens politiques d'une législation de la vie morale et d'une réforme des pratiques sociales. Avec la standardisation favorisée par les nouvelles conditions politiques et technologiques de la Confédération, le règne de Dieu dans la 
civilisation chrétienne d'une nation canadienne moderne et unifiée est devenu l'objectif concret auquel œuvraient les protestants voués à ce qu'on appellera le Social Gospel. L'esprit évangélique de réforme sociale importait plus que les énoncés théologiques et les structures canoniques, ce qui rendait possible les fusions ecclésiales successives censées mener à une seule Église pour l'État-nation du Canada, anglo-protestant, assimilateur et missionnaire : d'abord celle de tous les presbytériens en 1877 , suivie de celle des méthodistes en 1884 , jusqu'à ce que les uns et les autres rejoignent les congrégationalistes pour former l'Église unie du Canada en 1925 - date qui clôt la période couverte dans Early Presbyterianism in Canada, malgré la survivance d'une Église presbytérienne tronquée, que Moir a fini par rallier lui-même en 1972. Venu de l'Église unie, il se méfie en effet de l'étatisme (comparé au fascisme et au communisme) dans lequel est tombé le Social Gospel en confondant la fin et les moyens de l'avènement du Royaume de Dieu sur terre tout en perdant de vue Son image en chaque personne, ce qui appelle la réaction dans le sens opposé d'un fondamentalisme évangélique soucieux du seul salut individuel, signe de l'américanisation de la religion au Canada ( $C i C$, p. 7). N'empêche que le Social Gospel, sous des dehors progressistes (au racisme et au puritanisme près), s'inscrivait dans la tendance lourde « conservatrice » de l'histoire canadienne.

Ironically, the union of 1925 was also a return to the establishment principle - one nation, one church - that denominationalism had defeated almost a century earlier (CiC, p. 22).

In retrospect the Social Gospel was more preservative than revolutionary as it aimed to save the existing Christian Canada from the anti-religious and anti-Christian forces loosed against society by the industrial revolution (CiC, p. 21). 
Moir souligne régulièrement et à juste titre que le Social Gospel avait en ceci un exact pendant dans l'Action catholique, qui elle aussi cherchait à réaliser l'unité de la nation canadienne-française dans son cas - par la langue et pour la foi, en réponse aux effets dissolvants de la société industrielle. Par le relais de la League for Social Reconstruction et de la Révolution tranquille, leur influence continue d'ancrer dans une histoire religieuse aux sources largement contre-révolutionnaires les valeurs progressistes de solidarité sur lesquelles s'entendent les deux solitudes lorsqu'elles sont confrontées à l'option libérale américaine.

Today the values of Canada's Judeo-Christian heritage still inform the nation, and politicians employ such Social Gospel phrases as «the just society ». At home and abroad Social Gospel and Catholic Action ideals are proclaimed and practised by numerous Canadian organizations. Individually Canadians retain their passionate thirst for justice as the highest Christian virtue. They voice their concern for the welfare of others and their disapproval of discrimination against those less well-off (CiC, p. 23).

C'est là l'exemple préféré de John S. Moir pour montrer la nécessité d'intégrer la religion parmi les autres facteurs explicatifs de l'histoire canadienne tels que la politique et l'économie, et pour en appeler à un dépassement des clivages sectoriels en histoire, permettant de réaliser l'intrication de toutes ses facettes, comme il le fait admirablement dans ce choix d'écrits.

Quant à son élève Paul Laverdure, il répercute dans son livre nombre des problématiques chères à Moir, comme le déplacement par délégation ou par glissement de préoccupations à l'origine religieuse vers la sphère politique, où elles continuent d'agir souterrainement dans nos sociétés sécularisées. Mais son propos est d'abord de retracer à l'aide de leurs 
archives les efforts parallèles mais décalés de la Lord's Day Alliance et de la Ligue du dimanche, fers de lance respectifs du Social Gospel et de l'Action catholique, pour faire du Canada (tel que chacun de ces deux groupes l'entendait) une nation chrétienne par la digne observance du dimanche. Malgré d'importantes différences entre les protestants, qui révéraient dans le dimanche le saint sabbat de la Genèse, et les catholiques, qui ne voyaient en lui que le jour dévolu à la messe, son observance passait dans l'un et l'autre cas par la stigmatisation rhétorique, voire la répression judiciaire des réfractaires allogènes qui persistaient à faire des affaires ou à se distraire en public. Les Canadiens français s'étaient d'abord sentis visés par les dispositions puritaines du Lord's Day Act, dénoncé à la Chambre des Communes par Henri Bourassa comme l'atteinte d'une bigoterie médiévale à la liberté de la conscience individuelle d'appliquer la. loi morale. Les sabbatariens anglo-saxons furent ainsi interloqués de se voir taxer d'obscurantisme rétrograde par un papiste, certains qu'ils étaient d'être à la fine pointe du progrès social. Le Québec affirma même son caractère distinct sur ce point en votant, en remplacement de la loi fédérale de 1906, sa propre loi sur le sujet, qui avait la particularité de faire une exception pour les Juifs afin de tenir compte de leur sabbat, observé le samedi, en leur permettant de commercer le dimanche à la place.

Or, l'une des principales manifestations de l'Action catholique dans l'entre-deux-guerres, à l'instigation du Père Joseph-Papin Archambault, fut sa longue campagne pour une application rigoureuse de la loi du dimanche, souvent discutée dans l'historiographie de l'ère Taschereau. Le premier ministre dut se gagner l'appui du clergé en promettant de faire cesser le travail dominical dans l'industrie, mais n'obtint que des demi-mesures de ses amis capitalistes. Il crut bon de céder à la demande de sévir contre les cinémas suite à l'incendie meurtrier du Laurier Palace en 1927, mais se heurta à la résis- 
tance du public - son électorat. Devant ces échecs, la Ligue du dimanche se concentra donc de plus en plus sur les Juifs comme boucs émissaires, exigeant l'abrogation de l'exemption dont ils faisaient l'objet. Louis-Alexandre Taschereau usa de tous les prétextes pour se dérober à cette mesure, mais dut finalement accéder à ce désaveu d'une vieille conquête libérale et québécoise, et démissionna le lendemain. Maurice Duplessis dut ainsi, en partie, sa première victoire électorale à l'appui du clergé sur la base de sa promesse d'appliquer la loi, ce qu'il ne fit qu'à l'encontre de quelques petits commerçants, tandis que les usines ne s'arrêtèrent pas de tourner. Le dimanche cesserait d'ailleurs vite de mobiliser l'attention des critiques chrétiens de la société de consommation, qui n'aurait bientôt même plus d'oreilles pour les entendre; le jour du Seigneur y était devenu au mieux une vacance facultative, le « holy day » n'étant plus qu'une " holiday ». Laverdure aime à voir dans cette trace du sacré en son éclipse le signe du christianisme clandestin de la société canadienne, toujours prêt à resurgir sous des formes nouvelles; mais quoi qu'il en ait, cette interprétation spirituelle du congé hebdomadaire tel que vécu chez nous de nos jours pourra bien sembler tenir plutôt de l'acte de foi.

Christian Roy

Facultés de théologie et de philosophie Université Laval et Université de Sherbrooke 